\title{
Editorial
}

\section{Inhalation induction of anaesthesia}

The induction of sleep by inhalation has a tradition in anaesthesia which encompasses our very roots, as has the search for the ideal inhalation anaesthetic agent. Although rudimentary attempts at anaesthesia in earlier times have been described, many of us attribute the beginning of anaesthesia to Morton's demonstration of the inhalation of ether in the mid nineteenth century. Almost immediately, the search began for an agent which facilitated inhalation induction, as ether too often produced a slow, unpleasant and stormy induction. Chloroform was initially successful ${ }^{1}$ because it smelled more pleasing, was frequently associated with pleasant sensations, was rapidly taken up into the circulation, and caused the patient to pass quickly into sleep. Sleep was occasionally more permanent than planned; ventricular fibrillation in the early stages of anaesthesia sometimes ensued, and this complication, along with the hepatic necrosis which developed in selected patients, caused it to fall into disuse. Nitrous oxide, ${ }^{2}$ although used before ether, had to await the introduction of pressurized delivery systems to become popular. A very pleasant agent to inhale, nitrous oxide is a weak, albeit rapid-onset anaesthetic which cannot induce unconsciousness reliably except in hypoxic mixtures. Cyclopropane, an excellent gas for the induction of anaesthesia, has all but been abandoned because it is highly explosive. Halothane, an agent very similar in use to chloroform, never became established for inhalation induction in adults, possibly because the introduction of a rapid onset, short-acting intravenous anaesthetic agent preceded its design and synthesis.

Why did so many anaesthetists abandon inhalation induction, just as inhalation drug administration became popular with other specialists using drugs other than anaesthetics? Inhalational induction had been the standard for induction of anaesthesia for over one hundred years. Indeed, anaesthetists were the principal medical

From the Departments of Anaesthesia, Alberta Children's Hospital at the University of Calgary, and IWK-Grace Health Centre for Children*, Women and Families, Dalhousie University, Halifax. users of this route of drug administration, otherwise used by addicts of opium, cannabis, and tobacco. ${ }^{3}$ Inhalation induction of anaesthesia was abandoned because it was slow and smelly, caused excessive salivation, and resulted in coughing and vomiting. Intravenous anaesthesia had none of these problems. Even with the advent of halothane, many adults found its musty smell of thymolol to be malodourous, and induction slow. Inhalation induction did not therefore regain its initial popularity.

Intravenous induction of anaesthesia, although popular with adults, did not receive such widespread acceptance by children. Routine intravenous induction of anaesthesia for children is undesirable for some anaesthetists and for many patients (you don't have to be a child to know that needles hurt!). Halothane was a considerable advance, as it heralded an improvement on ether as an alternative to the intravenous induction of anaesthesia. Halothane has a relatively rapid onset, it smells nice (compared with ether), and it is not associated with vomiting during induction. Coughing and salivating are reduced. Halothane became the principal agent for inhalation induction of anaesthesia for many anaesthetists, and it has been a mainstay for anaesthesia in children for almost forty years.

Paediatric anaesthetists use many tricks, toys and innovations to facilitate inhalation induction of anaesthesia. Dr. McGraw has previously identified ${ }^{4}$ in the $C J A$ the psychological tools which should be used by anaesthetists in approaching a child prior to induction of anaesthesia. Indeed, the skills which he outlines are essential tools to use before any form of therapeutic intervention with a child. Parents are frequently asked, and are occasionally essential, to ease the induction of anaesthesia with a mask. Anaesthesia masks have improved remarkably in recent years, and many anaesthetists now use masks which are clear or transluscent, of multiple colours, and flavoured with the fruit essence of the child's choice. Flavoured extracts facilitate application of the anaesthetic mask, offering the child a choice of flavours allowing them some control over the process to which they are committed. Although "blowing up the balloon" is a favourite ploy of many, it some- 
times causes a child to focus on his breathing with resultant transient hyperventilation. This frequently leads to the arrest of respiration in the early stages of anaesthesia - a time when it is preferable to have the child breathing. Starting with $66 \%$ nitrous oxide before introducing any anaesthetic agent is a very useful beginning, as nitrous oxide is odour-free, its effects are frequently pleasant, and its presence improves tolerance of the added inhalation agent. Some paediatric anaesthetists add 5\% carbon dioxide to the inhaled anaesthetic mixture to increase minute ventilation thus speeding the uptake of anaesthetic agent, and with the use of anaesthetic agent monitors and capnometers, this is a much safer practice than it was in the past. Despite all these manouevres, inhalation induction of anaesthesia with halothane is not always smooth, and some patients continue to find it objectionable.

Isoflurane, enflurane, and desflurane have not improved either the comfort or safety of inhalation induction of anaesthesia. Airway complications occur more frequently with these drugs than they do with halothane. Coughing, breath-holding, and laryngospasm occur frequently with isoflurane, as confirmed by Cregg et al. in this issue of the CJA. The addition of humidification is evidently of no help in reducing the frequency and severity of laryngospasm. Although Cregg et al. ${ }^{5}$ should be credited for asking a simple question and using a simple but rigorous method to answer their question, the high frequency of airway complications in both of the groups they studied indicates that isoflurane is not the preferred agent for inhalation induction of anaesthesia in children. Both halothane and sevoflurane cause fewer airway complications.

Sevoflurane considerably improves the ease of inhalation induction. Recently introduced into the Canadian market, it is less irritating to the airway than either isoflurane or halothane, and is associated with fewer cardiac arrhythmias. ${ }^{6}$ In Japan, where sevoflurane has been available for several years, it has received widespread acceptance, and it is considered to be the most suitable agent (compared with halothane, isoflurane, and enflurane) for inhalation induction of anaesthesia. ${ }^{\text {? }}$

Sevoflurane results in more rapid induction of sleep and faster recovery from anaesthesia than does halothane, ${ }^{8-11}$ although improved speed of induction of surgical anaesthesia is not consistently born out in studies of induction times. In analyzing reports which compare the speed of induction with sevoflurane and other agents, the reader must bear in mind that a graduated increase in anaesthetic agent is no longer necessary with sevoflurane. Studies designed using a graduated increase in sevoflurane do not necessarily reflect clinical realities of use of the agent. Patients will readily accept $8 \%$ sevoflurane inspired concentration either as a single breath or once they are comfortable breathing $70 \%$ nitrous oxide, and anaesthetic agent uptake can be extremely rapid. It is our impression, both on the basis of clinical experience and reports in the literature, that the use of sevoflurane considerably improves the speed of inhalation induction of anaesthesia.

What are the disadvantages of sevoflurane? Concerns have been raised about its metabolism, its degradation in the presence of sodalime, the occurrence of excitation during induction, and cost. Metabolism of the drug to inorganic fluoride by hepatic cytochrome $\mathrm{P} 450$ has led to worries about possible nephrotoxicity. ${ }^{12}$ Although levels of fluoride have been transiently elevated following sevoflurane administration, increased levels have not been related to impairment of renal function, and they do not persist following anaesthesia. Post anaesthetic hepatitis occurs, but no more frequently than it does with enflurane or isoflurane. Sevoflurane undergoes degradation by sodalime ${ }^{13}$ in the presence of heat and moisture; this instability leads to the formation of Compound A. Either elimination of soda lime in the circuit will stop the production of Compound A, or induction of anaesthesia with high flow rates will dilute Compound $\mathrm{A}$ concentrations to clinically insignificant levels. Compared with halothane, sevoflurane causes an increased frequency of excitation during both induction $^{14}$ and recovery phases of anaesthesia. During induction, this is more frequent when sevoflurane is used in oxygen alone. The potential for the occurrence of excitement on induction should encourage the use of nitrous oxide and good practice of inducing anaesthesia in a quiet, warm, comfortable space without excessive noise, bright lights, or premature surgical stimulation. Excitement during recovery is due to the rapid emergence from anaesthesia before good analgesia is established. This emergence delirium can be minimized by careful attention to analgesia requirements and timing. It would seem that the most important disadvantage in using sevoflurane is cost: the present cost of sevoflurane is probably the greatest impediment to its more widespread use.

Not only does sevoflurane have advantages over other inhalation agents, it can also be used to supplant intravenous induction in suitable adult patients. In comparing the use of sevoflurane inhalation with the use of intravenous propofol in adults, sevoflurane has been shown to be an acceptable alternative for both induction and maintenance of anaesthesia ${ }^{15}$ - both propofol and sevoflurane have been shown to result in rapid induction, without significant side effects. ${ }^{16}$ Indeed, a single breath induction with sevoflurane has been shown to 
provide an acceptable alternative to intravenous induction in cooperative adult patients. ${ }^{17}$

Inhalation induction has a long and reputable history in anaesthesia, and sevoflurane makes inhalation induction a more attractive alternative for many patients. Perhaps the time has come to reevaluate exclusive reliance on intravenous induction for adults and consider offering inhalation induction to suitable patients. Although sevoflurane is not the ideal induction agent, it does bring us closer to our ideal and closer to our roots in inhalation induction of anaesthesia.

\section{L'induction par inhala- tion de l'anesthésie}

L'induction du sommeil par inhalation constitue une tradition qui fait partie du patrimoine de l'anesthésie et qui se perpétue par la recherche ininterrompue de l'agent d'inhalation idéal. Malgré tous les essais rudimentaires qui l'avaient précédée, pour plusieurs, l'anesthésie est née au milieu du dix-neuvième siècle avec la démonstration de l'inhalation de l'éther par Morton. Presque immédiatement après, on commençait à rechercher un nouvel agent capable de faciliter l'induction par inhalation pour remplacer l'éther dont l'induction était lente, désagréable et orageuse. Le chloroforme a connu ses heures de gloire ${ }^{1}$ parce qu'il sentait bon, parce qu'il était associé à des sensations agréables et parce qu'il pénétrait plus rapidement dans la circulation assurant ainsi un sommeil plus rapide. À l'occasion, le sommeil atteignait une permanence indésirable; une fibrillation ventriculaire survenait parfois aux premiers stages de l'anesthésie et cette complication, comme la nécrose hépatique, a hâté sa désuétude. Bien qu'il ait été utilisé avant l'éther, le protoxyde d'azote ${ }^{2}$ a dû attendre l'introduction de systèmes d'administration pressurisés pour devenir populaire. Très agréable à respirer, le protoxyde d'azote est un agent faible, tout en étant rapide d'action, qui ne peut induire l'inconscience de façon fiable excepté si on l'administre à des concentrations hypoxiques. Le cyclopropane, un gaz excellent pour l'induire l'anesthésie, a dû être abandonné à cause de ses propriétés fortement explosives. L'halothane, qui ressemble beaucoup au chloroforme ne s'est jamais imposé avec fermeté chez les adultes comme agent d'induction par inhalation, possiblement parce que les agents intra- veineux à début rapide et à courte durée d'action l'ont précédé.

Pourquoi tant d'anesthésistes ont-ils abandonné l'induction par inhalation alors que dans d'autres disciplines l'administration par inhalation de médicaments prenait de l'ampleur? L'induction par inhalation n'avaitelle pas été en anesthésie la norme pendant plus de cent ans? Les anesthésistes étaient vraiment les principaux utilisateurs de cette voie d'administration si on fait exception des habitués de l'opium, du cannabis et du tabac. ${ }^{3}$ En réalité, l'induction par inhalation a été abandonnée parce qu'elle était lente, déplaisante pour l'odorat, faisait saliver excessivement et provoquait de la toux et des vomissements. L'anesthésie intraveineuse ne comportait aucun de ces inconvénients. Même avec l'arrivée de l'halothane, l'odeur du thymolol et la lenteur de son induction répugnaient aux adultes. Ainsi, l'induction par inhalation n'a jamais pu retrouver sa vogue d'antan.

Par contre, même si elle était très appréciée par les adultes, l'induction intraveineuse n'a jamais été, en règle générale, appréciée par les enfants. L'induction intraveineuse systématique était rejetée par certains anesthésistes et par de nombreux patients (il n'est pas nécessaire d'être un enfant pour constater que les aiguilles sont douloureuses!). L'halothane constituait un pas en avant parce que cet agent correspondait à une amélioration sur l'éther si on désirait éviter l'induction intraveineuse. L'halothane a un début d'action relativement rapide, sent bon (comparativement à l'éther) et n'est pas associé aux vomissements pendant l'induction de l'anesthésie. La toux et la salivation sont moins importantes. Pour plusieurs anesthésistes, pendant près de 40 ans, l'halothane est demeuré l'agent d'induction incontournable pour l'anesthésie des enfants.

Les anesthésistes pédiatriques utilisent plusieurs subterfuges, jouets et innovations pour faciliter l'induction par inhalation. Le docteur McGraw a déjà identifé ${ }^{4}$ dans notre journal les ressources psychologiques que devraient utiliser l'anesthésiste lorsqu'il est temps d'induire l'anesthésie chez l'enfant. En réalité, les astuces qu'il décrit sont des techniques qui doivent précéder toute forme d'intervention thérapeutique chez l'enfant. On demande fréquemment aux parents de participer à l'induction de l'anesthésie au masque; ce concours peut même être quelquefois essentiel. Ces dernières années, les masques se sont considérablement améliorés; des masques clairs ou translucides de différentes couleurs et aromatisés à une essence de fruit que l'enfant choisit, sont maintenant utilisés par plusieurs anesthésistes. Les extraits aromatiques facilitent l'application du masque, en offrant à l'enfant un choix d'essences qui lui donne l'impression d'exercer une sorte de contrôle sur le pro- 
cessus qu'il doit supporter. Bien que « souffler dans le ballon " soit une astuce utilisée par plusieurs, cette méthode porte parfois l'enfant à se concentrer sur sa respiration ce qui l'amènera à s'hyperventiler momentanément. Ceci peut provoquer l'arrêt de la respiration aux stages initiaux de l'anesthésie, un moment où il est nécessaire de respirer. Comme le protoxyde d'azote n'a pas d'odeur, débuter avec cet agent à $66 \%$ avant d'introduire l'agent anesthésique constitue une approche efficace qui permet d'ajouter plus facilement l'agent anesthésique principal. Quelques anesthésistes pédiatriques ajoutent $5 \%$ de gaz carbonique au mélange inhalé dans le but d'augmenter la ventilation minute et d'accélérer l'absorption de l'anesthésique; grâce aux moniteurs de concentration et aux capnomètres, cette méthode constitue une pratique plus sûre que dans le passé. Malgré toutes ces manoeuvres, l'induction par inhalation à l'halothane ne se fait pas toujours sans heurts et certains patients continuent de s'y objecter:

L'isoflurane, l'enflurane et le desflurane n'ont amélioré ni le confort ni la sécurité de l'induction de l'anesthésie par inhalation. Les complications qui affectent les voies aériennes sont plus fréquentes avec ces agents qu'avec l'halothane. La toux, l'apnée et le laryngospasme surviennent souvent avec l'isoflurane, comme le confirment Gregg et al. dans ce numéro du JCA. De toute évidence, l'humidification n'aide pas à réduire la fréquence et la gravité du laryngospasme. Bien que Gregg et al. ${ }^{5}$ aient eu le mérite de poser une question simple et d'utiliser une méthode simple et rigoureuse pour y répondre, la fréquence élevée des complications des voies aériennes dans le deux groupes à l'étude montre que l'isoflurane n'est pas l'agent de choix pour l'induction de l'anesthésie par inhalation des enfants. L'halothane, comme le sévoflurane est moins irritant pour les voies respiratoires.

Le sévoflurane permet une induction beaucoup mieux réglée. Récemment introduit au Canada, le sévoflurane et moins irritant pour les voies respiratoires que 1 'isoflurane et l'halothane tout en étant moins arythmogène. ${ }^{6}$ Au Japon, où le sévoflurane est universellement adopté, cet agent est considéré comme le plus approprié pour l'induction par inhalation (lorsque comparé à l'halothane, l'isoflurane et l'enflurane). ${ }^{7}$

Le sévoflurane procure une induction et un réveil plus rapides que l'halothane, ${ }^{8-11}$ bien que l'accélération de la vitesse d'induction jusqu'au stade chirurgical ne soit pas démontrée dans toutes les études réalisées sur ce sujet. En consultant les comptes rendus qui comparent la vitesse d'induction du sévoflurane avec d'autres agents, le lecteur doit avoir à l'esprit qu'avec cet agent, l'augmentation progressive de la concentration n'est plus justifiée. Les études basées sur l'augmentation graduelle du sévoflurane vont à l'encontre des avantages procurés par cet agent. Les patients acceptent d'emblée une concentration inspirée de $8 \%$ de sévoflurane administrée en une seule inspiration ou une fois après quelques inspirations de protoxyde d'azote à 70\%; l'absorption de l'agent anesthésique se fait alors très rapidement. L'expérience clinique comme la littérature nous incite à croire que l'utilisation du sévoflurane améliore sensiblement la vitesse de l'induction.

Quels sont les désavantages du sévoflurane? Son métabolisme, sa dégradation au contact de la chaux sodée, l'agitation qu'il provoque à l'induction et son coût. Son métabolisme en fluor inorganique par le cytochrome hépatique $\mathrm{P} 450$ faisait appréhender une néphrotoxicité éventuelle. ${ }^{12}$ Toutefois, bien que la concentration de fluor s'élève temporairement pendant l'administration du sévoflurane, cette augmentation n'a pas provoqué de dysfonctionnement rénal et ne persiste pas après l'anesthésie. L'hépatite postanesthésique peut survenir, mais pas plus souvent qu'avec l'enflurane ou l'isoflurane. Le sévoflurane est dégradé par la chaux sodée $^{13}$ en présence de chaleur et d'humidité; cette instabilité conduit à la formation du Composé A. L'élimination de la chaux sodée du circuit annule la production du Composé A; l'induction de l'anesthésie avec des grands débits ramène par dilution la concentration du Composé $\mathrm{A}$ à des niveaux inoffensifs. Comparativement à l'halothane, le sévoflurane augmente la fréquence des épisodes d'agitation aussi bien à l'induction $^{14}$ qu'à la phase de récupération. Pendant l'induction, cet inconvénient arrive plus souvent lorsque le sévoflurane est utilisé en oxygène pur. La possibilité d'excitation à l'induction devrait encourager l'association du protoxyde d'azote; il est aussi sage d'induire l'anesthésie dans un endroit calme, chauffé et confortable, sans bruits excessifs, lumières aveuglantes et sans stimulation chirurgicale prématurée. Au réveil, l'excitation résulte de ce que l'émergence de l'anesthésie se produit avant l'installation de l'analgésie. Ce délire d'émergence peut être minimisé si on porte une attention particulière au début et à la qualité de l'analgésie. Il semble que la désavantage le plus important du sévoflurane soit son coût actuel, lequel constitue vraisemblablement un obstacle majeur à la généralisation de son emploi.

En plus de sa supériorité sur les autres agents d'inhalation, le sévoflurane peut remplacer avantageusement, chez certains adultes, l'induction intraveineuse. En comparant l'inhalation du sévoflurane avec l'administration intraveineuse de propofol chez l'adulte, on a démontré que le sévoflurane pouvait procurer une solution de rechange acceptable autant pour l'induction que pour le maintien de l'anesthésie, ${ }^{15}$ ces deux agents étant 
tous deux capables d'induire l'anesthésie rapidement et sans effets secondaires importants. ${ }^{17}$

L'induction par inhalation a une longue et remarquable histoire; le sévoflurane représente pour certains patients une solution très attrayante. Le moment est peut-être venu de réévaluer chez l'adulte notre dépendance exclusive envers l'induction intraveineuse et de considérer l'induction par inhalation à l'occasion. Bien que le sévoflurane ne soit pas l'agent idéal d'induction, il s'en approche et perpétue nos traditions fondées sur l'induction de l'anesthésie par inhalation.

\section{References}

1 Simpson JY. Account of a new anaesthetic agent, as a substitute for sulphuric ether in surgery and midwifery. In: Cole F (Ed.). Milestones in Anesthesia. Lincoln: University of Nebraska Press, 1965: 92-101.

$2 O^{\prime}$ Connor JP. Nitrous oxide 1844-1990. Can J Anaesth 1990; 37: 603-7.

3 Gravenstein JS. The history of drug inhalation: a brief overview. Anesth Analg 1980; 59: 140-5.

4 McGraw T. Preparing children for the operating room: psychological issues. Can J Anaesth 1994; 41: 1094-103.

5 Cregg N, Wall C, Green D, Mannion D, Casey W. Humidification reduces coughing and breath-holding during inhalation induction with isoflurane in children. Can J Anaesth 1996; 43: 1090-4.

6 Johannesson GP, Florén M, Lindahl SGE. Sevoflurane for ENT-surgery in children. A comparison with halothane. Acta Anaesthesiol Scand 1995; 39: 546-50.

7 Doi $M$, Ikeda $K$. Airway irritation produced by volatile anaesthetics during brief inhalation: comparison of halothane, enflurane, isoflurane and sevoflurane. Can J Anaesth 1993; 40: 122-6.

8 Black A, Sury MRJ, Hemington L, Howard R, Mackersie $A, H a t c h D J$. A comparison of the induction characteristics of sevoflurane and halothane in children. Anaesthesia 1996; 51: 539-42.

9 Greenspun JCF, Hannallah RS, Welborn LG, Norden JM. Comparison of sevoflurane and halothane anesthesia in children undergoing outpatient ear, nose, and throat surgery. J Clin Anesth 1995; 7: 398-402.

10 Sury MRJ, Black A, Hemington L, Howard R, Hatch DJ, Mackersie A. A comparison of the recovery characteristics of sevoflurane and halothane in children. Anaesthesia 1996; 51: 54j-6.

11 Lerman J, Davis PJ, Welborn LG, et al. Induction, recovery, and safety characteristics of sevoflurane in children undergoing ambulatory surgery. Anesthesiology 1996; 84 : 1332-40.

12 Brown B Jr. Sevoflurane: introduction and overview. Anesth Analg 1995; 81: S1-3.
13 Strum DP, Johnson BH, Eger EI II. Stability of sevoflurane in soda lime. Anesthesiology 1987; 67: 779-81.

14 Sarner JB, Levine M, Davis PJ, Lerman J, Cook DR, Motoyama EK. Clinical characteristics of sevoflurane in children. Anesthesiology 1995; 82: 38-46.

15 Fredman B, Nathanson MH, Smith I, Wang J, Klein K, White $P F$. Sevoflurane for outpatient anesthesia: a comparison with propofol. Anesth Analg 1995; 81: 823-8.

16 Smith I, Ding $Y$, White $P F$. Comparison of induction, maintenance, and recovery characteristics of sevoflurane$\mathrm{N}_{2} \mathrm{O}$ and propofol-sevoflurane- $\mathrm{N}_{2} \mathrm{O}$ with propofol-isoflurane- $\mathrm{N}_{2} \mathrm{O}$ anesthesia. Anesth Analg 1992; 74: 253.9.

17 Fukuda H, Kasuda H, Saitoh $K$, et al. Rapid induction of anesthesia with inhalation of sevoflurane. (Japanese) Masui 1993; 42: 1744-7. 